\title{
Commissioning and Operation Experience with the CEBAF Recirculation Arc Beam Transport System*
}

\author{
Y. Chao, M. Crofford, N. Dobeck, D. Douglas, A. Hofler, C. Hovater, G. A. Krafft, \\ R. Legg, J. Perry, E. Price, S. Suhring, M. Tiefenback, and J. van Zeijts \\ CEBAF, 12000 Jefferson Avenue, Newport News, Va. 23606
}

\section{Abstract}

Results of the initial beam tests and early operation of the CEBAF recirculation beam transport system are presented.

\section{INTRODUCTION AND SCOPE OF TESTS}

From October 1992 to April 1993, pre-commissioning checkout and beam tests of the CEBAF accelerator were conducted. This paper documents the scope and results of these tests and relates experience gained.

The CEBAF superconducting accelerator is a recirculating $\mathrm{cw}$ electron linac consisting of a $45 \mathrm{MeV}$ injector linac, two $0.4 \mathrm{GeV}$ main linacs, and a recirculator. The design is unchanged from previous reports [1], and comprises lattices of five subsystems: injector, linacs, spreader/recombiners, extraction regions, and semicircular arcs. Here, we describe initial tests of the system through the $135^{\circ}$ point of the lowest energy arc. The subsystem tested consisted of the injector and north linac lattices, the lowest energy spreader and extraction transport lines, and three of the four superperiods of the lowest energy east arc recirculation transport line.

Accelerator beam operations resumed on 23 November 1992 after an extended construction-related shutdown. By 4 December, $135 \mathrm{MeV}$ beam had been brought to the end of the spreader, and on 11 December, beam was taken to a dump at the $135^{\circ}$ point of the low energy east arc beam line. Testing continued until 9 April 1993.

Tests conducted during this period fell into three categories. Firstly, algorithms for setup and tuning of the transport system were developed. Secondly, comparisons were made between observed machine behavior and that predicted by design and modeling of the system. Finally, descrepancies between modeling and observations were detailed, and possible reconciliations tested.

\section{SETUP AND TUNING}

Numerous procedures for accelerator setup and tuning were developed during this running period. These are designed to utilize values from machine modeling and previous accelerator runs, and, with a minimum of operator intervention, propagate well-tuned beam losslessly through the accelerator. We now describe the major algorithms.

\section{A. Betatron Phase Space Matching}

At the end of the injector and following the spreader, betatron phase matching regions allow for a match of the beam ellipse from the upstream transport to the acceptance of the downstream beamline. This is accomplished by making an emittance measurement, which specifies the betation parameters at the front end of the phase matching region (see section IIE). These values are used as initial conditions for a DIMAD computation of quadrupole excitations required to provide the desired betatron match. Simple downloading of the resulting quadrupole values,

\footnotetext{
"Supported by D.O.E. contract \#DE-AC05-84ER40150
}

with no additional trimming of quadrupole strengths, produced no observable betatron mismatch through either a well tuned linac or arc. A measurement of beam properties at the end of the linac implies agreement of observed and computed betatron functions to the 20-30\% level, which is consistent with the error of measurement in the initial values provided by the emittance measurement.

\section{B. Linac FODO Tuning}

The linac $120^{\circ}$ FODO lattice is tuned using an algorithm in which betatron oscillations are excited by steering magnets and driven to nodes every third half-cell by quadrupoles. The resulting optics has phase advances with absolute errors of $2-5^{\circ}$ along the length over which the nodal pattern is constructed; no betatron mismatch is evident in the beam spots down the linac. Use of this procedure gives agreement in phase advance, quadrupole excitation curves, and beam energy, to a level of $1-2 \%$. The distance over which the procedure is applicable is limited by horizontal/vertical coupling induced by RF cavities; this is discussed in more detail in section IIID.

\section{Spreader Dispersion Suppression}

Following the linac, the spreader is dispersion suppressed by using two vertically focussing quadrupoles $1 / 4$ betatron wavelength apart to create dispersion nodes at beam position monitors that are, respectively, $1 / 4$ and $11 / 2$ betation wavelengths from the second quad. The transport is achromatic and has a momentum acceptance of $\sim 3 \%$; the measurement is described in section IIF. The quadrupole excitations required for good dispersion suppression were noted to be $5-10 \%$ different from those predicted by the machine model and quadrupole excitation curves. This discrepancy is now understood to be due to end fields in transport system dipoles.

\section{Adjustment of Arc Momentum Compaction}

In section IV, we describe tests of isochronicity in this system. Here, we note that the momentum compaction is adjustable over a broad range by varying a pair of quadrupoles, a half-wavelength apart, in each arc superperiod. This was successfully performed as a part of the isochronicity measurement described below, and serves as a first step in the development of algorithms to tune simultaneously arc phase advances, dispersion, and momentum compaction.

In summary, the testing of setup and tuning algorithms resulted in successful procedures for machine operation. No undue system sensitivities were encountered, and any discrepancies were attributable to errors in quadrupole excitation curves (which are now resolved to the 1-2\% level) and/or dipole end fields (which are now being understood quantitatively). The resulting algorithms behave in accordance with observation and modeling, and can be readily automated. Two major procedures remain to be developed. The first will provide fine-tuning of phase matches between machine modules; the second will provide detailed control of arc transport parameters. Both will depend on applications of difference orbit techniques presently under development (see section IIIE). 
We note that these tests were carried out at 120-135 $\mathrm{MeV}$, which is $1 / 4$ to $1 / 3$ the design energy. The energy offset was readily accommodated by application of measured dipole and quadrupole excitation curves at lower currents, and by careful attention to standardizing hysteresis loops, to avoid errors due to magnet remnant fields.

We will now discuss a pair of measurements that were frequently performed as a part of setup and for verification of proper beamline tuning.

\section{E. Emittance/Momentum Spread Measurements}

Emittance measurements were performed to obtain $\beta$ function values for use in matching computations. Table 1 summarizes typical results obtained at the injector (20 $\mathrm{MeV})$, the arc $(121 \mathrm{MeV}, 10 \mu \mathrm{A})$, and the spreader entrance $(121 \mathrm{MeV}, 36 \mu \mathrm{A})$.

In general, measurements were performed in a regime (pulsed mode, low current) that does not represent beam characteristics at design operating conditions; however, the observed $\beta$ functions agree with design values. The measurement is estimated to resolve betatron function values in excess of $10 \mathrm{~m}$ to $20-30 \%$ and values under $5 \mathrm{~m}$ to a factor of two.

\section{Table 1: Emittance Data}

$\begin{array}{lllll}\begin{array}{l}\text { Energy } \\ (\mathrm{MeV})\end{array} & \begin{array}{l}\epsilon_{x} \\ (\text { nm-rad) }\end{array} & \begin{array}{l}\epsilon_{y} \\ (\text { nm-rad) }\end{array} & \begin{array}{l}\text { Average } \\ \text { Current } \\ (\mu \mathrm{A})\end{array} & \begin{array}{l}\text { Macropulse } \\ \text { Width } \\ (\mu \mathrm{sec})\end{array} \\ 20 & 10.8 & 8.2 & 10 & 150 \\ 121 & 0.7 & 1.8^{*} & 10 & 150 \\ 121 & 0.7 & - & 36 & 100\end{array}$

*We note that the value $\epsilon_{y}=1.8 \mathrm{~nm}$-rad measured in the recirculator was corrupted by residual dispersion and should be regarded as an upper bound.

The value $\epsilon_{x}=0.7 \mathrm{~nm}$-rad measured in the arc is adiabatically damped from the $10.8 \mathrm{~nm}$-rad value observed in the injector. An emittance measurement performed at a higher current indicated that $\epsilon_{x}$ values remained consistent with values obtained at lower currents. A measurement of energy spread conducted in conjunction with the high current $\epsilon_{x}$ measurement yielded $\sigma_{\delta p / p}=9 \times 10^{-5}$ [2].

Horizontal-vertical coupling [3] was a detriment to this measurement. Discrepancies in the 20 and $121 \mathrm{MeV}$ emittances (which should adiabatically vary by only a factor of 6) may be due to this. Coupling was alleviated when the injector was well tuned.

\section{F. Dispersion Measurements}

Detailed dispersion measurements were made during difference orbit measurements; a separate vertical dispersion measurement indicated good agreement with design. Residual and error dispersions were nominally at the $10 \mathrm{~cm}$ level. As noted, spreader momentum acceptance is $\sim 3 \%$.

\section{COMPARISON OF DESIGN, MODELING, AND OBSERVATION}

Tests of the above procedures provided opportunities to compare design and simulation predictions against observation. Points of comparison were thereby established.

\section{A. Quadrupole Excitation Curves}

Quadrupole excitation curves from magnetic measurment were verified by tuning of the linac FODO lattice to $\sim 1 \%$. Hysteresis effects were important, as remnant fields could change quad excitation at low energies by $50 \%$ or more. Standardized quadrupole cycling avoided errors of this type.

\section{B. Dipole Excitation Curves}

Excitation curves were verified by spreader and arc orbit setup to $\sim 1 / 2 \%$ if hysteresis effects were ignored; agreement between magnetic-measurement-based predictions and observeration was estimated to be better than $0.1 \%$ if magnets were operated on a standard hysteresis loop.

\section{Dipole End Fields}

Effects of end fields in arc dipoles were observed. Behavior was consistent with measurement and modeling [4]. The focussing effect of the end fields of a dipole was equivalent, in the arc, to trim of $1-3 \%$ of arc quads; in spreader magnets (with larger sagitta), the effect was equivalent to trims of $2-10 \%$ of dispersion suppressor quads. This is consistent with observation (see sections IIC and IV); these effects will be included in improvements to the machine model.

\section{Horizontal/Vertical Coupling}

RF-cavity-generated coupling was observed in the injector and linac, and, when evident, limited the distance over which linac FODO tuning was possible to approximately 1 to $11 / 2$ betatron wavelengths. Behavior was consistent with simulation of cavity coupling effects [5].

Horizontal/vertical coupling was not observable in the arcs provided the orbit was well corrected (residual of a few millimeters) though arc dipoles. If beam steering was poor (residual of several millimeters), dipole end-field-generated coupling could be observed.

As noted above, this experience suggested two possibly useful tuning algorithms that are yet to be developed: fine tuning of phase matching regions and fine tuning simultaneously of arc phase advances, dispersion and momentum compaction. Development of these procedures will depend on use of difference orbit measurements for detailed understanding of the optics.

\section{E. Difference Orbit Measurements}

Detailed verification of lattice properties can be obtained through use of difference orbit measurement techniques. A program is therefore under development to measure transfer matrices through the system using BPM readings of trajectories with different launch conditions covering the 5-dimensional phase space $\left(x, x^{\prime}, y, y^{\prime}, \delta p / p\right)$. Localized sections of beamline are used as a basis for inferring global optical behavior. This difference orbit method has been employed successfully in measurements elsewhere [6].

The program includes procedures for determining the full linear transfer matrix. This is done by utilizing BPM readings of specific orbit offsets. Injection conditions can be established by fitting to these readings, with BPM reading errors being established from separate measurements. A number of features in the program allow for flexible operation. For example, algorithms allow for the use data from an arbitrary number of BPMs in the fitting process, along with the exact propagation of errors. In addition, defective data are eliminated by an iterative screening process. All contributions to the final error analysis, including BPM errors, signal/noise ratio in every set of trajectories, as well as errors incurred during the nonlinear fitting procedure, are propagated to the final transfer matrix elements. This yields information on both the nonlinear optical behavior in the system and on the accuracy of the optics setup in the local sections. 
Test versions of this program were used to measure the optics in the spreader and east arc. Preliminary results imply that the divergence in the rms BPM data is consistent with the expected deviations due to nonlinear optical effects and the measured transfer matrix elements. This method of insuring proper optical behavior is expected to be increasingly useful as the program evolves; it will become the principal tool for checking lattice behavior and verifying proper beamline tuning during next running period.

\section{MEASUREMENT OF ISOCHRONICITY}

A major test of design and modeling against observation was provided by a measurement of the $M_{58}$ linear transfer matrix element from the front end of the east arc spreader to the $90^{\circ}$ point. This was done using a modified version of the phase transfer measurement detector [7] (Figure 1), and provides a test of the isochronicity and tuneablity of the transport system. A $1500 \mathrm{MHz}$ precision phase detector is used to measure the phase difference between a reference signal derived from one of the final RF control modules in the linac and an amplified beam signal originating in the BPM at the $90^{\circ}$ point. The variable phase shifter is used to calibrate the measurement; drifts are negligible on the measurement time scale. Simultaneously a modulator changes the linac energy.

\section{Precision Phase Detector}

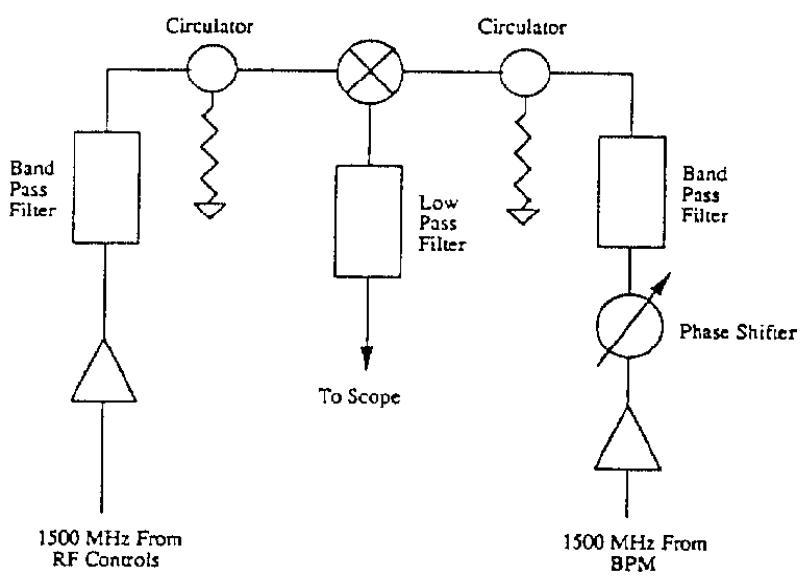

Figure 1. Schematic for $M_{56}$ measurement.

Two optics sets were calculated using DIMAD. The first was the nominal isochronous arc tuning, with a nominal $M_{58}$ of $0 \mathrm{~m}$. The second was a special tuning with a nominal $M_{56}$ of $-1 \mathrm{~m}$, and was generated from the first by varying the excitation of a specific family of arc quadrupoles (which, by design, control $M_{56}$ ) by $15 \%$ while trimming the 3 remaining families to restore the design tunes and dispersion suppression. The special tuning was measured to have an $M_{56}$ of absolute magnitude $1.4 \mathrm{~m}$; the deviation from design is equivalent to a quadrupole excitation error of $6 \%$ in the family used to control momentum compaction, which, together with the effects of dipole end fields, is consistent with the type of error observed in other tests. The nominal tuning was measured to have an $M_{56}$ of $18.5 \mathrm{~cm}$. Small corrections of $1-2 \%$ were iteratively applied to the compaction control quadrupoles while $M_{50}$ was repeatedly measured. After these quads were shifted by an average of $3 \%$, the $M_{58}$ was measured to be $1.8 \mathrm{~cm}$. This deliberately generated shift in $M_{56}$ is consistent with the $0.4 \mathrm{~m}$ per $6 \%$ quad excitation offset observed above, and is also consistent with the effect of dipole end fields (section IIIC).

This technique can probably be used to measure $M_{50}$ to an accuracy of better than $3 \mathrm{~mm}$, as compared to the presently estimated resolution of $\sim 1 \mathrm{~cm}$.

\section{FUTURE DIRECTIONS}

The experience gained during this testing period suggests a number of additions to machine hardware, control, and operating procedures that will be useful during the next running period. Regular use of difference orbit measurements will be made to verify machine tuning and resolve discrepancies between design and observed behavior. Incorporation of measured dipole end-field effects into machine modeling will improve the robustness of tuning cormputations; algorithms for fine-tuning of module-to-module betatron phase matches and for arc tuning are under development. Installation of shims on low energy arc dipoles will reduce the magnitude of end field effects.

Hysteresis effects influence system reproducibility. Automated procedures for standardizing magnets at startup and after machine tuning will therefore be implemented, and will insure that magnet excitations remain on a standard cycle. The RF vernier system will provide control of linac energy drifts by stabilizing both gradient and phase [8]. Additional control system utilities, including automated beam threading and steering as well as tuning of transport line focusing properties, will assist in machine setup [9].

Improvements will be made in emittance measurement techniques, which at present are limited by profile monitor cycle time. These could be implemented through simultaneous use of multiple harps, and/or use of beam steering across a fixed wire profile monitor. Finally, an analog monitoring system is being implemented, which will allow access to various useful signals, such as output from beam loss monitors distributed around the machine.

In summary, this test period has provided confirmation of many design choices. The transport system behaves predictably and in general accordance with design and modeling. Many automated procedures are under development, and guidance has been obtained for the resolution of outstanding questions.

\section{REFERENCES}

[1] B. Bowling et al., "The CEBAF Beam Transport System Lattice Design", Proc. 1991 IEEE Part. Acc. Conf., San Francisco, CA, May, 1991, pp. 446-448.

[2] Work performed in collaboration with $\mathrm{L}$. Merminga and members of the CEBAF High Power Test Team.

[3] M. Tiefenback et al., "Emittance Measurements and Transverse Cavity Transfer Matrix in the CEBAF Nuclear Physics Accelerator", these proceedings.

[4] D. Douglas, "The Effect of Observed Dipole End Fields On Beam Transport System Optics", CEBAFTN-91-095, 10 December 1991.

[5] Z. Li et al., "Transport Properties of the CEBAF Cavity", these proceedings; M. Tiefenback et al., op. cit.

[6] T. Barklow, SLAC-PUB-4969; T. Barkow, P. Emma, and $N$. Walker, unpublished SLAC seminars.

[7] C. G. Yao, "A Method to Measure Phase Distribution of Charge in Bunch", CEBAF-TN-0131, $20 \mathrm{June} 1989$.

[8] G. A. Kraft et al., "Energy Vernier System for CEBAF", these proceedings.

[9] B. Bowling et al., "Orbit Correction Implementation at CEBAF", these proceedings. 